\title{
Análise de indicadores ambientais no reservatório do Passaúna
}

\section{Analysis of environmental indicators in Passaúna reservoir}

\author{
Jorge Mattar Neto \\ Mestre pelo Programa de Mestrado em Gestão Ambiental da Universidade Positivo \\ Cláudio Marchand Krüger \\ Coordenador do Curso de Engenharia Civil da Universidade Positivo \\ Maurício Dziedzic \\ Coordenador do Programa de Mestrado em Gestão Ambiental da Universidade Positivo
}

\section{Resumo}

A importância dos indicadores ambientais está associada à sua utilização como instrumentos para a sociedade avaliar a própria evolução. No contexto ambiental, indicadores são parâmetros representativos, concisos e fáceis de interpretar utilizados para ilustrar as principais características de uma determinada região. O presente trabalho apresenta uma proposta de indicadores ambientais para o reservatório do Passaúna, um dos principais mananciais de Curitiba, com base em 14 parâmetros. Apresenta, também, uma proposta de determinação de um índice de comprometimento ambiental (ICA) com base na importância relativa de cada um dos potenciais poluidores. Apresenta-se, aqui, um exemplo de determinação do ICA, considerando os dados disponíveis para seis parâmetros, concluindo-se que o reservatório do Passaúna apresenta forte comprometimento ambiental.

Palavras-chave: sustentabilidade; indicador ambiental; reservatório do Passaúna.

\section{Abstract}

The relevance of environmental indicators is associated with their use as tools with which society evaluates its own evolution. In the environmental context, indicators are parameters which are representative of the processes involved, are concise and easy to interpret, and which are used to represent the main characteristics of a certain area. The present work proposes a set of environmental indicators for the Passaúna reservoir, one of the main sources of drinking water in Curitiba, Brazil, based on 14 parameters. This paper also presents a proposal for the determination of an environmental stress index (ICA) based on the relative importance of pollution threats. An example is shown, considering the available data for six parameters, which indicates that the Passaúna reservoir is under strong environmental stress.

Keywords: sustainability; environmental indicator; Passaúna reservoir.

\section{Introdução}

A partir da década de 1980, o mundo começou a demonstrar sua preocupação com as questões ambientais e surgiram reuniões de âmbito mundial para discussão dos problemas do meio ambiente. A busca por soluções encontra sua direção no conceito de desenvolvimento sustentável, que deve enfatizar a nova consciência ambiental da sociedade globalizada. A constatação do problema atual alerta para os problemas futuros, como bem descreve Camargo (2003):

Se um dos mais importantes avanços do século XX foi o despertar de uma consciência ambiental e da necessidade de encontrar um equilibrio entre as ações humanas e a preservação do meio ambiente onde vivemos, os desafios para o século XXI relacionados à busca de soluções para nossos graves e globais problemas socioambientais serão, contudo, muito mais complexos e profundos, uma vez que já há sinais evidentes de uma crise de insustentabilidade ecológica e social que se arma em todo o planeta. (p.14).

A demanda pelos limitados recursos naturais está sempre crescendo e, portanto, torna-se imprescindível a tomada de decisões de forma cuidadosa, bem como a verificação do impacto do crescimento na utilização desses recursos. A poluição do ar, dos mares e rios, o efeito estufa, a diminuição da camada de ozônio e o comprometimento dos recursos hídricos são alguns dos problemas ambientais 
decorrentes do aumento da população mundial e hoje fazem parte das discussões em todo o mundo.

Dentre os problemas ambientais que o crescimento populacional nos grandes centros exacerba, o comprometimento das bacias hidrográficas merece uma atenção especial:

Durante a urbanização, os espaços permeáveis, inclusive áreas vegetadas e bosques, são convertidas para o uso que, geralmente, provoca o aumento de áreas com a superficie impermeável, resultando no aumento de volume do escoamento superficial e da carga de poluentes. (ARAÚJO; ALMEIDA; GUERRA, 2005, p. 36).

Hoje, o desenvolvimento econômico e social não pode ser considerado sem que a preocupação ambiental esteja presente em todo o processo, configurando os indicadores ambientais como instrumentos de planejamento e gestão dos espaços urbanos e rurais. Tendo um conjunto de indicadores como ferramentas para o planejamento, haverá parâmetros para um melhor aproveitamento dos recursos naturais e também para a indicação de medidas preventivas de degradação ambiental e consequentes prejuízos econômicos.

A importância dos indicadores ambientais está associada à sua utilização como um instrumento que a sociedade terá para avaliar seu progresso, sua evolução ou ainda um instrumento de planejamento e gestão dos espaços urbanos e rurais. O conjunto de indicadores ambientais servirá para propiciar um melhor aproveitamento dos recursos naturais e também para indicação de medidas preventivas de degradação ambiental e consequentes prejuízos econômicos para a sua reparação. A informação que liga fatores socioeconômicos e ambientais oferece uma sólida base empírica para construir indicadores de sustentabilidade e, dessa maneira, medir os impactos e avaliar a eficiência sob vários pontos de vista dos processos de desenvolvimento.

A fim de possibilitar que o desenvolvimento sustentável se torne operacional, necessitam-se indicadores ambientais como instrumentos usados na busca de parâmetros dessa sustentabilidade. Portanto, em relação ao reservatório do Passaúna, o presente estudo colabora na tomada de decisão para gerenciar e minimizar problemas que envolvem o uso do reservatório e a garantia da conservação, bem como a melhoria da qualidade ambiental da água e da região de influência.

\section{Desenvolvimento sustentável}

Desenvolvimento sustentável, segundo definição dada no Relatório da Comissão Mundial de Meio Ambiente e Desenvolvimento, é aquele que atende às demandas da geração presente sem comprometer as oportunidades atuais e futuras.

A aparente simplicidade desta definição envolve reflexões devido a inter-relações atreladas ao desenvolvimento, demandas e oportunidades atuais e futuras, bem como todos os aspectos sociais, econômicos, culturais e ambientais no momento presente e também no futuro, tanto no âmbito global como nos regionais e locais (MALHEIRO; ASSUNÇÃO, 2000 apud BOOG; BIZZO, 2003).

$\mathrm{Na}$ Conferência das Nações Unidas sobre Meio Ambiente e Desenvolvimento, realizada em 1992, no Brasil, conhecida como Rio-92, o desenvolvimento sustentável foi a tônica das discussões e se confirmou como fator primordial para atingir um nível de excelência na relação entre homem e meio ambiente. Propostas de ações que quantificavam, avaliavam e monitoravam as condições ambientais das regiões foram definidas no encontro, resultando na Agenda 21, documento que demonstra a preocupação da sociedade com os problemas ambientais.

O desenvolvimento sustentável almejado pela sociedade requer uma regulamentação do processo de oferta e procura ambiental, através de problemas gerados por desequilíbrios, ocupação e uso desordenado do solo urbano. Promover mudanças nos padrões de produção e consumo da cidade, reduzindo custos e desperdícios e fornecendo o desenvolvimento de tecnologias urbanas sustentáveis, bem como desenvolver e estimular a aplicação de instrumentos econômicos no gerenciamento dos recursos naturais visando à sustentabilidade urbana são estratégias que devem ser utilizadas.

Para Capra (1996), o paradigma atual está numa visão holística, que concebe o mundo como um todo integrado e não como uma coleção de partes dissociadas.

Nessa nova visão, que ele denomina "Ecologia Profunda", o mundo não é uma coleção de objetos isolados, mas uma rede de fenômenos fundamentalmente interconectados e interdependentes.

Dessa forma os indicadores constituem componentes de avaliação ambiental importantes, capazes de quantificar alterações na qualidade do meio ambiente e quantidade de recursos naturais, bem como de avaliar os esforços desenvolvidos visando à melhoria do meio ambiente ou a mitigação de sua degradação.

\section{Indicadores ambientais}

Um indicador, segundo a Agência Europeia de Meio Ambiente (AEMA), é um valor representativo de um fenômeno que quantifica a informação mediante a agregação de diferentes dados, dando lugar à informação sintetizada. Os indicadores simplificam a informação ajudando a descrever e valorar fenômenos mais complexos (VIEIRAS; POULIQUEN; SOTO, 2005).

Ou ainda:

Os indicadores podem comunicar ou informar sobre o progresso em direção a uma determinada meta, como, por exemplo, o desenvolvimento sustentável, mas também podem ser entendidos 
como um recurso que deixa mais perceptível uma tendência ou fenômeno que não seja imediatamente detectável. (HAMMOND et al, 1995 apud BELLEN, 2005, p. 41).

Os indicadores de sustentabilidade constituem um sistema de sinais que permitem aos países avaliar seu progresso em gestão ambiental a respeito do desenvolvimento sustentável. Os indicadores ambientais, assim como os econômicos e sociais, permitem que se compare uma base comum de informação selecionada e processada, o que facilita a objetividade nos processos de decisão, seu ordenamento, geração e enriquecimento mediante o fortalecimento da participação dos cidadãos (QUIROGA, 2005).

Para se chegar a um indicador capaz de mensurar processos não-sustentáveis de desenvolvimento, é necessário estabelecer um conjunto de critérios objetivos e verificáveis como, por exemplo: ser significativo para a avaliação do sistema; ter validade, objetividade e consistência; ter coerência e ser sensível a mudanças no tempo e no sistema; ser de fácil mensuração, baseado em informações facilmente disponíveis e de baixo custo; permitir a relação com outros indicadores (DEPONTI; ECKERT; AZAMBUJA, 2002).

Merico (1997 apud RUFINO, 2002) destaca que os indicadores ambientais são usados para se ter um retrato da qualidade ambiental e dos recursos naturais, além de avaliar as condições e as tendências ambientais rumo ao desenvolvimento sustentável.

A escolha dos indicadores ambientais é fator preponderante para sua posterior aplicação. As propriedades que devem caracterizar os indicadores, segundo a Organização para a Cooperação e Desenvolvimento Econômico (OCDE) (1993, apud ALMEIDA; BRITO, 2002), são:

- relevância: deve ser representativo, de fácil compreensão e comparável;

- consistência: deve ser bem apoiado em termos técnicos e científicos e de consenso internacional;

- mensurabilidade: deve ser facilmente mensurável e passível de monitoramento regular a um custo não excessivo.

A necessidade de criação e desenvolvimento de indicadores de sustentabilidade fica confirmada no workshop denominado Indicators for sustainable development for decision making (GHENT, 1995 apud BELLEN, 2005):

A utilidade dos indicadores de sustentabilidade, como mencionado na Agenda 21, foi confirmada pelo workshop. Os usos potenciais desses sistemas incluem o alerta aos tomadores de decisão para as questões prioritárias, orientação na formulação de políticas, simplificação e melhora na comunicação e promoção do entendimento sobre tendências-chave fornecendo a visão necessária para as iniciativas de ação nacional. (p. 53).
A utilização dos Indicadores Ambientais torna-se importante para os decisores na medida em que as atitudes do ser humano refletem no meio ambiente, formando cadeias de ação-resposta e permitindo uma verificação dos impactos causados:

Constata-se a existência de um ciclo, começando com uma pressão humana sobre o meio ambiente, afetando de forma diferente os diversos segmentos sociais e, termina, com uma resposta da sociedade. (Cunha, 2005, p. 4).

Ainda não se tem um modelo ideal que possibilite mensurar os impactos ambientais. Existem, porém, opções disponíveis para seleção e desenvolvimento de indicadores, como os modelos Esquema para o Desenvolvimento de Estatísticas Ambientais (EDEA) e PressãoEstado-Resposta (PER). Os mais usados são os modelos para a tomada de decisão ou elaboração de estratégias que definem a relação entre a informação ambiental e os valores sociais e/ou objetivos e metas políticas; e modelos dos processos ambientais e das interações sociedade/ meio ambiente que tratam de classificar os problemas ambientais em termos de causa/efeito (OCDE, 1993 apud CUNHA, 2005).

Apesar dos avanços obtidos por alguns países, ainda existem obstáculos a serem vencidos de forma mais harmônica e produtiva. Nesse sentido, desenvolver estudos sobre os indicadores se faz necessário diante da urgente necessidade de fortalecimento da capacidade dos países de produzir estatísticas sociais e ambientais confiáveis que permitam uma melhor formulação e monitoramento das políticas de desenvolvimento (QUIROGA, 2005).

\section{Metodologia}

O presente trabalho caracteriza-se como um estudo de caso, um estudo empírico e que investiga um fenômeno atual dentro do contexto real. Tem lugar definido como um método de pesquisa, pois entre várias aplicações, pode-se citar a que descreve a situação real do contexto no qual está sendo feita a intervenção (PATTON, 1980 apud DONAIRE, 1997). Neste caso, trata-se do Reservatório do Passaúna, localizado a oeste da região metropolitana de Curitiba, envolvendo os municípios Almirante Tamandaré, Araucária, Campo Largo, Campo Magro e Curitiba.

Tendo como ponto inicial o Relatório da Área de Proteção Ambiental (APA) do Passaúna (SANEPAR, 2001), efetuou-se uma análise dos indicadores de sustentabilidade sugeridos por organizações como: Associação para a Defesa Ecológica de Galícia (Adega), Comissão de Desenvolvimento Sustentável (CDS) da Organização das Nações Unidas (ONU) e Instituto Brasileiro de Geografia e Estatística (IBGE). A partir dessa análise selecionou-se um grupo de indicadores ambientais que representasse o comprometimento do meio ambiente no reservatório do Passaúna e, em seguida, elaborou-se uma proposta de determinação de um índice de mensuração do comprometimento ambiental para o local. 


\section{Fundamentação teórica}

Em busca de uma forma simples de mensurar o possível comprometimento ambiental da região e usando indicadores que privilegiassem a fácil leitura e compreensão do fenômeno, foi proposta uma forma de calcular um índice que representasse o comprometimento da região.

Com base na presença de potenciais poluidores nos municípios, calcula-se o Índice de Comprometimento Ambiental (ICA) de cada indicador referente a cada um dos setores (área do município inserido na região de estudo) e, em seguida, obtém-se o ICA médio desse indicador para a região.

O cálculo do ICA é feito usando-se a Equação 1, que é uma definição de concentração relativa. A região corresponde à área total, que é subdividida em setores.

$$
\mathrm{ICA}_{\text {setor }}=\frac{\frac{\mathrm{Q}_{\mathrm{S}}}{\mathrm{A}_{\mathrm{S}}}}{\frac{\mathrm{Q}_{\mathrm{R}}}{\mathrm{A}_{\mathrm{R}}}}
$$

onde:

$\mathrm{Q}_{\mathrm{R}}$ : quantidade referente ao potencial de poluição da região de estudo;

$\mathrm{Q}_{\mathrm{S}}$ : quantidade referente ao potencial de poluição no setor;

$A_{R}$ : área da região de estudo;

$\mathrm{A}_{\mathrm{S}}$ : área do setor.

Em seguida, é obtido o ICA da região pela soma dos produtos envolvendo o ICA médio de cada indicador e respectivo potencial de degradação $\left(\mathrm{w}_{\mathrm{i}}\right)$. A Equação 2 descreve o cálculo:

$$
\operatorname{ICA}_{\text {região }}=\sum_{\mathrm{i}=1}^{\mathrm{n}} \mathrm{ICA}_{\text {médio }} \cdot \mathrm{w}_{\mathrm{i}}
$$

onde:

$\mathrm{ICA}_{\text {médio }}$ : ICA médio dos indicadores na região;

$\mathrm{w}_{\mathrm{i}}$ : potencial de degradação do indicador.

O potencial de degradação $\left(\mathrm{w}_{\mathrm{i}}\right)$ de cada indicador em relação ao conjunto de indicadores é obtido aplicando-se o método de Mudge, utilizado na análise de valor. O método permite classificar hierarquicamente os indicadores, baseando-se numa análise comparativa entre os mesmos, dois a dois, até que todos sejam comparados entre si (BARROS, 2002).

Tabela 1 - Classificação da escala de potencial de degradação

\begin{tabular}{lc} 
Potencial de degradação & Classificação \\
\hline Muito baixo & 1 \\
\hline Baixo & 2 \\
Médio & 3 \\
\hline Forte & 4 \\
Muito forte & 5
\end{tabular}

O método de Mudge é uma técnica que consiste em comparar todas as possíveis combinações de pares de funções, determinando-se a cada momento a mais importante, com uma ponderação adequada. Terminadas as comparações e somados os pontos de cada função na matriz, tem-se a função de maior importância relativa seguida pelas demais (CSILLAG, 1995).

A Tabela 1 apresenta uma escala de degradação, onde o valor 1 representa um grau muito baixo e 5 um grau muito forte de degradação.

Depois de calcular o ICA da região, obtém-se um índice ajustado

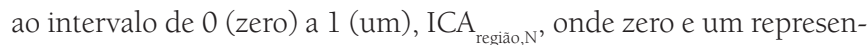
tam, respectivamente, menor e maior comprometimento ambiental. O cálculo é feito através da Equação 3.

$$
\mathrm{ICA}_{\text {região, }}=\frac{\mathrm{ICA}_{\text {geral }}-\mathrm{ICA}_{\text {min }}}{\mathrm{ICA}_{\text {máx }}-\mathrm{ICA}_{\text {min }}}
$$

onde:

ICA $_{\text {regiáo }}$ : ICA da região em estudo;

$\mathrm{ICA}_{\min }$ : menor ICA possível da região;

$\mathrm{ICA}_{\text {máx: }}$ maior ICA possível da região.

O ICA ${ }_{\min }$ é obtido pelo cálculo do ICA para a região com concentração de potenciais poluidores na maior área, resultando em 0,56; o $\mathrm{ICA}_{\max }$ é obtido pelo cálculo do ICA para a região com concentração de potenciais poluidores na menor área, resultando em 1,73 para este caso.

Com o ICA $A_{\text {regiào, }}$ pode-se classificar o comprometimento da região utilizando-se a escala de intensidade de comprometimento apresentada na Tabela 2.

O Índice do Estado Trófico (IET) é utilizado no cálculo do ICA. Trata-se de um índice simples e prático usado para auxiliar o estudo e o gerenciamento da qualidade dos recursos hídricos. Os valores de transparência da água obtidos pelo disco de Secchi são transformados pela Equação 4 em índice de estado trófico para a profundidade de Secchi - IET(DS), e os dados referentes a Clorofila são transformados em índice do estado trófico para a clorofila - IET(CL) pela Equação 5 (CARLSON, 1977).

$$
\begin{aligned}
& \operatorname{IET}(\mathrm{DS})=10 \cdot\left(6-\frac{\ln \mathrm{DS}}{\ln 2}\right) \\
& \operatorname{IET}(\mathrm{Cl})=10 \cdot\left(6-\frac{2,04-0,68 \cdot \ln \mathrm{Cl}}{\ln 2}\right)
\end{aligned}
$$$$
\text { Equação } 4
$$

Tabela 2 - Escala de intensidade de comprometimento

$\begin{array}{lc}\text { ICA }_{\text {regiáo,N }} & \text { Intensidade de comprometimento } \\ 0 \text { a } 0,25 & \text { Fraca } \\ 0,26 \text { a } 0,50 & \text { Moderada } \\ 0,51 \text { a } 0,75 & \text { Forte } \\ 0,76 \text { a } 1,00 & \text { Muito forte }\end{array}$


onde:

DS: profundidade medida com o disco de Secchi em metros;

$\mathrm{Cl}$ : concentração de clorofila-A medida à superfície da água, em $\mu \mathrm{g} . \mathrm{L}^{-1}$;

ln: logaritmo natural.

\section{Conjunto de indicadores ambientais na APA do Passaúna}

A determinação de ICA é fundamentada na concentração dos potenciais poluidores por setor e também pela importância relativa de cada indicador na composição do índice. Esses setores são definidos pelos municípios inseridos na APA do Passaúna (Curitiba, Campo Largo e Araucária).

Os indicadores foram selecionados levando-se em conta sua importância e disponibilidade, e estão relacionados na Tabela 3. Estes foram classificados segundo seu potencial de degradação e identificados por letras com objetivo de construir a matriz de Mudge. A classificação foi estabelecida levando-se em conta os graus da escala de potencial de degradação (Tabela 1) e a análise de cada indicador.

A classificação dos potenciais de degradação pode ser determinada após uma sondagem (tipo survey) (GIL, 2002) entre especialistas na área ambiental, buscando um consenso de opiniões que servirá de parâmetro para o estabelecimento dos pesos utilizados no presente estudo. Cabe ressaltar que neste estudo o intuito é apresentar a metodologia de análise, e a classificação apresentada reflete a percepção dos autores em relação a cada indicador especificamente no reservatório do Passaúna, não tendo significado absoluto, mas apenas para o problema analisado. Para utilização da mesma metodologia em outros estudos de caso, tanto a seleção dos indicadores quanto a sua classificação, devem ser objeto de análise cuidadosa.

A construção da matriz de Mudge, Tabela 4, é feita com a distribuição dos indicadores de forma escalonada: o primeiro indicador na segunda linha e primeira coluna; o segundo, na terceira linha e segunda coluna; o terceiro, na quarta linha e terceira coluna, e assim sucessivamente até que todos os indicadores fossem colocados. Na primeira linha da matriz também são colocados os indicadores, respeitando a ordem de distribuição inicial, a partir do segundo, para que a comparação dois a dois pudesse ser feita.

A comparação é feita avaliando-se o potencial de degradação de cada um, e o indicador de maior potencial de degradação entra na matriz com um índice obtido pela diferença entre o potencial de degradação dele com o do outro indicador. Assim, o indicador identificado por A (População prevista) apresenta potencial de degradação 4 (Forte), maior do que o do indicador B (Pontos de acesso) com potencial de degradação 3 (Médio); a diferença entre eles é o índice que o indicador A levará para a matriz, A1. No caso em que a diferença é nula, ambos se equivalem no potencial de degradação e, nesse caso, a escolha fica a cargo do pesquisador.

Após completar a matriz de Mudge, o potencial de degradação de cada indicador em relação aos demais é obtido pela soma dos índices da letra associada às linhas da matriz, mais um (por exemplo, linha $\mathrm{A}: \mathrm{A} 1+\mathrm{A} 1+\mathrm{A} 3+\mathrm{A} 2+\mathrm{A} 3+\mathrm{A} 2+\mathrm{A} 1+\mathrm{A} 2+\mathrm{A} 2=17+1=18) \cdot \mathrm{A}$ coluna percentual (\%) indica a importância relativa de degradação do indicador com relação aos outros - 10\%, usada como fator de ponderação no cálculo do ICA região.

\section{Cálculo do índice de comprometimento ambiental (ICA) no reservatório do Passaúna}

Desenvolve-se a seguir, para fins de ilustração, um exemplo de aplicação do ICA na concentração dos pontos de acesso ao reservatório do Passaúna, fontes de poluição, a população prevista na região em estudo com base nos loteamentos aprovados na área em estudo, o déficit de oxigênio dissolvido (DOD), IET obtido a partir da presença de clorofila e o IET obtido a partir da profundidade Secchi.

A Tabela 5 apresenta a área de cada município inserida da região de estudo e os dados referentes aos sete indicadores escolhidos para o cálculo.

Tabela 3 - Lista de indicadores ambientais e classificação do potencial de degradação

\begin{tabular}{|c|c|c|}
\hline Indicador & Identificação & Classificação \\
\hline População prevista & A & 4 \\
\hline Pontos de acesso & $\mathrm{B}$ & 3 \\
\hline Fontes de poluição & $\mathrm{C}$ & 5 \\
\hline Consumo de água & $\mathrm{D}$ & 3 \\
\hline Área urbana & $E$ & 5 \\
\hline Fitoplâncton & $\mathrm{F}$ & 1 \\
\hline Agrotóxicos & G & 4 \\
\hline Coliformes & $\mathrm{H}$ & 4 \\
\hline Déficit de oxigênio dissolvido (DOD) & I & 2 \\
\hline Índice de estado trófico IET (Clorofila) & $\mathrm{J}$ & 1 \\
\hline Massa total de partículas & $\mathrm{K}$ & 2 \\
\hline $\begin{array}{l}\text { Índice de estado trófico IET } \\
\text { (Disco de Secchi) }\end{array}$ & $\mathrm{L}$ & 3 \\
\hline Tratamento de esgoto & $M$ & 2 \\
\hline
\end{tabular}


Tabela 4 - Matriz de valoração de Mudge referente ao potencial de degradação dos indicadores selecionados

\begin{tabular}{|c|c|c|c|c|c|c|c|c|c|c|c|c|c|c|c|c|}
\hline & B & C & D & $E$ & $F$ & $\mathrm{G}$ & $\mathrm{H}$ & I & $\mathrm{J}$ & $\mathrm{K}$ & $\mathrm{L}$ & $M$ & $N$ & $\mathrm{Pi}^{\star}$ & $\Sigma$ & (\%) \\
\hline \multirow[t]{15}{*}{ A } & $\mathrm{A} 1$ & C1 & $\mathrm{A} 1$ & $\mathrm{E} 1$ & $\mathrm{~A} 3$ & $\mathrm{G} 1$ & $\mathrm{H} 1$ & A2 & A3 & A2 & $\mathrm{A} 1$ & A2 & A2 & 1 & 18 & 10 \\
\hline & $B$ & C2 & B1 & E2 & B2 & $\mathrm{G} 1$ & $\mathrm{H} 1$ & B1 & B2 & B1 & L1 & B1 & B1 & 1 & 10 & 6 \\
\hline & & C & $\mathrm{C} 2$ & $\mathrm{C} 1$ & C4 & $\mathrm{C} 1$ & $\mathrm{C} 1$ & C3 & C4 & C3 & C2 & C3 & C3 & 1 & 31 & 18 \\
\hline & & & D & E2 & D2 & $\mathrm{G} 1$ & $\mathrm{H} 1$ & D1 & D2 & D1 & L1 & D1 & D1 & 1 & 9 & 5 \\
\hline & & & & E & $\mathrm{E} 4$ & $\mathrm{E} 1$ & E1 & E3 & E4 & E3 & E2 & E3 & E3 & 1 & 30 & 18 \\
\hline & & & & & $F$ & G3 & $\mathrm{H} 3$ & 11 & J1 & K1 & L2 & M1 & N1 & 1 & 1 & 1 \\
\hline & & & & & & G & G1 & G2 & G3 & G2 & G1 & G2 & G2 & 1 & 20 & 12 \\
\hline & & & & & & & $\mathrm{H}$ & $\mathrm{H} 2$ & H3 & $\mathrm{H} 2$ & $\mathrm{H} 1$ & $\mathrm{H} 2$ & $\mathrm{H} 2$ & 1 & 19 & 11 \\
\hline & & & & & & & & I & $\mid 1$ & 11 & L1 & 11 & N1 & 1 & 5 & 3 \\
\hline & & & & & & & & & $\mathrm{J}$ & K1 & L2 & M1 & N1 & 1 & 2 & 1 \\
\hline & & & & & & & & & & $\mathrm{K}$ & L1 & M1 & N1 & 1 & 3 & 2 \\
\hline & & & & & & & & & & & L & L1 & L1 & 1 & 11 & 7 \\
\hline & & & & & & & & & & & & $M$ & N1 & 1 & 4 & 2 \\
\hline & & & & & & & & & & & & & $\mathrm{N}$ & 1 & 6 & 4 \\
\hline & Poten & degr & ão ini & & & & & & & & & & & $\mathrm{T}$ & 169 & 100 \\
\hline
\end{tabular}

Tabela 5 - Valores utilizados na determinação do ICA

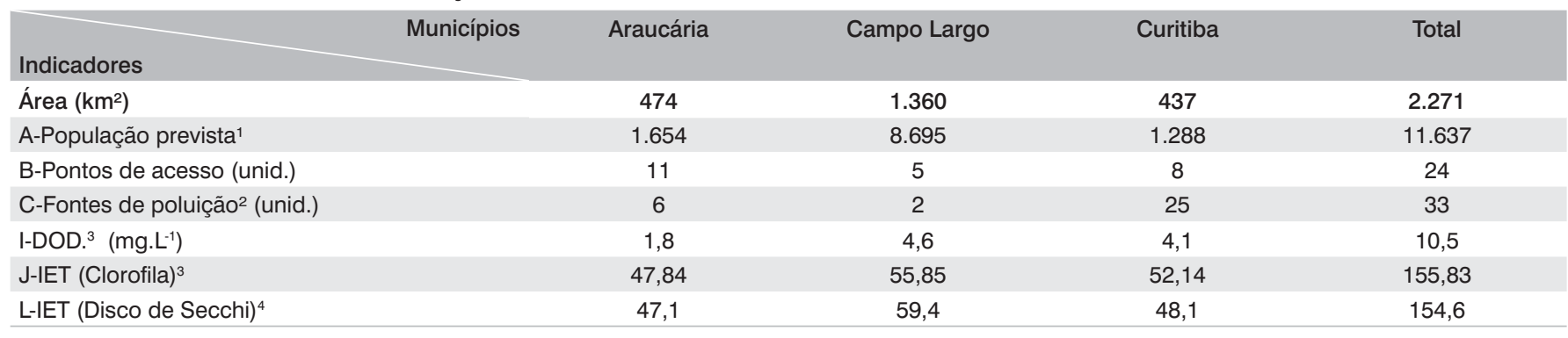

Fonte: Sanepar (2001) e Meger (2007).

1 População prevista com base no número de lotes dos loteamentos aprovados na APA e na média da RMC de 3,7 habitantes por unidade (lotes); ${ }^{2}$ indústrias, cemitérios, pedreiras e lixões. ${ }^{3}$ valores médios do IET obtidos a partir da presença de Clorofila, de Meger (2007); ${ }^{4}$ valores médios do IET obtidos a partir da profundidade Secchi, de Meger (2007).

O primeiro passo é calcular um índice para cada setor utilizando a Equação 1 e, em seguida, obter a média dos três setores.

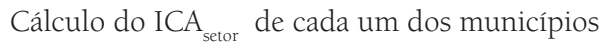

População prevista:

$\underline{1654}$

$\underline{474}$

- Araucária $\rightarrow \mathrm{ICA}_{\text {pop,Arau }}=\underline{11637} \cong 0,68$

2271

$\underline{8695}$

$\underline{1360}$

- Campo Largo $\rightarrow \mathrm{ICA}_{\text {pop, }, \mathrm{CL}}=\underline{11637} \cong 1,25$

2271

1288

$\underline{437}$

- $\quad$ Curitiba $\rightarrow \mathrm{ICA}_{\text {pop,Ctba }}=$
- Índice médio da População Prevista: $\mathrm{ICA}_{\text {médio }}=\underline{0,68+1,25+0,58} \cong 0,84$

3

Pontos de acesso:

$\begin{array}{ll} & \underline{11} \\ & \underline{474} \\ \underline{24} \cong 2,20 \\ & 2271 \\ & \underline{5} \\ & \underline{1360} \\ & \underline{24} \cong 0,35 \\ & 2271 \\ & \\ \text { - Campo Largo } \rightarrow \mathrm{ICA}_{\mathrm{pts}, \mathrm{CL}}= & \underline{8} \\ & \underline{437} \\ & \underline{24} \cong 1,73 \\ & 2271\end{array}$


- Índice médio dos pontos de acesso:

$\mathrm{ICA}_{\text {médio }}=\underline{2,20+0,35+1,73} \cong 1,43$ 3

Fontes de poluição:

6

- $\quad$ Araucária $\rightarrow \mathrm{ICA}_{\mathrm{fp}, \text { Arau }}=$

$\underline{474} \cong 0,87$

$\underline{33}$

2271

$\underline{2}$

- Campo Largo $\rightarrow \mathrm{ICA}_{\mathrm{fp}, \mathrm{CL}}=\underline{1360} \cong 0,10$

$\underline{25}$

- Curitiba $\rightarrow \mathrm{ICA}_{\mathrm{fp}, \mathrm{Ctba}}=$ $\underline{437} \cong 3,94$

- Índice médio das Fontes de Poluição:

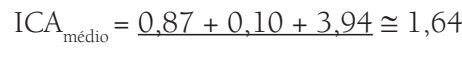
3

Déficit de oxigênio dissolvido:

- $\quad$ Araucária $\rightarrow \mathrm{ICA}_{\text {dod,Arau }}=$

$\underline{474} \cong 0,82$

10,5

$\underline{4,6}$

- Campo Largo $\rightarrow \mathrm{ICA}_{\text {dod,CL }}=$

$\underline{1360} \cong 0,73$

$\underline{10,5}$

2271

$\underline{4,1}$

- $\quad$ Curitiba $\rightarrow$ ICA $_{\text {dod,Ctba }}=$

- Índice médio do déficit de oxigênio dissolvido: $\mathrm{ICA}_{\text {médio }}=\underline{0,82+0,73+2,03} \cong 1,20$ 3

Índice Estado Trófico (clorofila):

- $\quad$ Araucária $\rightarrow \mathrm{ICA}_{\text {clor Arau }}=$ $\underline{55,85}$

- Campo Largo $\rightarrow \mathrm{ICA}_{\text {clor, }, \mathrm{CL}}=\underline{1360} \cong 0,60$

$\underline{155,83}$

2271

$\underline{52,14}$

- Curitiba $\rightarrow$ ICA $_{\text {clor,Ctba }}=$

- Índice médio da clorofila: $\mathrm{ICA}_{\text {médio }}=1,47+0,60+1,74 \cong 1,27$

3

Índice Estado Trófico (disco de Secchi):

- $\quad$ Araucária $\rightarrow \mathrm{ICA}_{\mathrm{IET}, \mathrm{Ctba}}=$ $\underline{47.1}$

474 $\cong 1,46$

$\underline{154,6}$

2271

$\underline{59,4}$

- Campo Largo $\rightarrow \mathrm{ICA}_{\mathrm{IET}, \mathrm{Ctba}}=\underline{1360} \cong 0,64$

$\underline{154,6}$

2271

$\underline{48,1}$

- Curitiba $\rightarrow \mathrm{ICA}_{\mathrm{IET}, \mathrm{Ctba}}=$

$\underline{437} \cong 1,62$

$\underline{154,6}$

2271

- Índice médio do estado trófico:

$$
\mathrm{ICA}_{\text {médio }}=\underline{1,46+0,64+1,62} \cong 1,24
$$

3

\section{Classificação do potencial de degradação de cada indicador}

Em seguida, determina-se a classificação de cada indicador envolvido no cálculo utilizando-se a Tabela 1. Os indicadores do exemplo estão classificados na Tabela 6 .

A construção da matriz de Mudge (Tabela 7) é feita comparandose os indicadores dispostos em linha com os indicadores colocados nas colunas em relação ao potencial de degradação de cada indicador. Após completar a matriz de Mudge, o potencial de degradação relativo de cada indicador em relação aos demais aparece na coluna percentual (\%).

\section{Cálculo do ICA da região}

Considerando os resultados obtidos na Tabela 7, observa-se que Fontes de Poluição (C) é o indicador com o maior potencial de degradação em relação aos outros cinco do exemplo com um percentual de 34\%; a presença de clorofila (J) é o que tem o menor potencial com 
Tabela 6 - Classificação do potencial de degradação dos indicadores usados no exemplo

\begin{tabular}{lcc|}
\hline Indicador & Identificação & Classificação \\
\hline População prevista & A & 4 \\
\hline Pontos de acesso & B & 3 \\
\hline Fontes de poluição & C & 5 \\
\hline DOD & I & 2 \\
\hline IET (CL) & J & 1 \\
IET (DS) & L & 3 \\
\hline
\end{tabular}

3\%, o cálculo do índice geral de comprometimento ambiental fica assim definido usando a Equação 2:

$\mathrm{ICA}_{\text {região }}=0,84.0,24+1,43.0,21+1,64.0,34+1,20.0,06+1,27.0,03+1,24.0,12$

$\mathrm{ICA}_{\text {região }} \cong 1,32$

Visando classificar a região quanto ao grau de comprometimento ambiental apresentado na Tabela 2, calculou-se um índice ajustado ao intervalo de 0 (zero) a 1 (um) a partir da Equação 3.

O ICA geral normalizado $\left(\mathrm{ICA}_{\text {regiào,N }}\right)$ fica igual a:

$$
\mathrm{ICA}_{\text {região,N }}=\frac{1,32-0,56}{1,73-0,56} \quad \therefore \quad \mathrm{ICA}_{\text {regão, }} \cong 0,65
$$

\section{Análise dos resultados}

Com base na análise dos indicadores: Pontos de Acesso, Fontes de Poluição, População Prevista, DOD, IET (CL) e IET (DS); a região tem um índice de comprometimento ambiental de 0,65 que, segundo a classificação apresentada na Tabela 2, é considerado forte, o que indica a necessidade de uma resposta rápida da sociedade, sendo necessárias medidas urgentes para que a degradação ambiental na região seja minimizada.

\section{Conclusão}

Após análise, constatou-se que o índice de comprometimento ambiental da região estudada é igual a 0,65, conforme a tabela de escala de intensidade de comprometimento (Tabela 2). Essa medida pertence ao intervalo de 0,51 a 0,75 , que representa uma classificação de comprometimento ambiental forte. A classificação forte sugere a necessidade de monitoramento sistemático e frequente da região.
Tabela 7 - Matriz de valoração de mudge referente ao potencial de degradação dos indicadores usados para a determinação do ICA geral

\begin{tabular}{ccccccccc} 
& B & C & I & J & L & Pi $^{*}$ & $\sum$ & $(\%)$ \\
A & A1 & C1 & A2 & A3 & A1 & 1 & 8 & 24 \\
& B & B2 & B1 & B2 & B1 & 1 & 7 & 21 \\
& & C & C3 & C4 & C2 & 1 & 11 & 34 \\
& & I & I1 & L1 & 1 & 2 & 6 \\
& & & J & L2 & 1 & 1 & 3 \\
& & & & L & 1 & 4 & 12 \\
& & & & & Total & 33 & 100 \\
\hline
\end{tabular}

*Potencial de degradação inicial

$\mathrm{Na}$ análise dos indicadores, comprovou-se que a presença do homem na região é fator preponderante de comprometimento ambiental, pois entre os indicadores selecionados, fontes de poluição e área urbana aparecem com a maior importância relativa no que diz respeito ao potencial de poluição. Ressalta-se que essas análises são resultados de um exemplo da aplicação da proposta e que, para se obter resultados mais consistentes, os dados devem ser referenciados todos a um mesmo período, o que não é verdadeiro para o exemplo apresentado. Todavia, já pode ser tomado como indicação da situação vigente.

A presente proposta tem a intenção de tornar simples a análise ambiental e fornecer subsídios para a discussão e gerenciamento ambiental. A metodologia proposta se justifica como pertinente, na identificação de parâmetros, para a avaliação e a elaboração de planejamento ambiental e na tomada de decisões que minimizem os problemas de comprometimento na região.

O desenvolvimento da metodologia fundamenta-se no cálculo da concentração de poluidores em setores de uma dada região e no potencial de degradação relativo de cada um. Ao utilizar o método de Mudge para analisar e classificar os potenciais poluidores de uma região, possibilita-se uma seleção crítica de indicadores para a composição de um índice geral de qualidade ambiental.

A partir do presente trabalho, a determinação do índice de qualidade ambiental pode ser aprimorada inserindo-se, por exemplo, uma análise de dispersão dos dados referentes aos setores pertencentes à região estudada. Para uma aplicação mais consistente, de maneira a fornecer subsídios conclusivos para a gestão ambiental da região, a adoção da metodologia deve ser acompanhada da coleta sistemática dos dados necessários dos respectivos indicadores.

Finalmente, deve-se ressaltar que a determinação do ICA de uma região caracteriza-se como um instrumento capaz de contribuir para avaliar a evolução do processo de planejamento e gestão ambiental em busca do desenvolvimento sustentável. 


\section{Referências}

ALMEIDA, J.; BRITO, A.G. A utilização de indicadores ambientais como suporte ao planejamento e gestão de recursos hídricos: o caso da região autônoma dos açores. In: III CONGRESO IBÉRICO SOBRE GESTIÓN Y PLANIFICACIÓN DEL ÁGUA, Anais..., Sevilla, Portugal, novembro de 2002.

ARAUJO, G.H.S.; ALMEIDA, J.R.; GUERRA, A.J.T. Gestão ambiental de áreas degradadas. Rio de Janeiro: Bertrand Brasil, 2005.

BARROS, M.M.N. Incorporando a melhoria contínua em áreas de suporte visando à qualidade global em serviços: uma proposta de modelo para melhoria da qualidade nas ações das áreas de suporte. Dissertação (Mestrado em Engenharia de Produção) - Universidade Federal de Santa Catarina, Florianópolis, 2002.

BELLEN, H.M. Indicadores de sustentabilidade: uma análise comparativa. Rio de Janeiro: FGV, 2005

BOOG, E.G.; BIZZO, W.A. A utilização de indicadores ambientais como instrumento para gestão de desempenho ambiental em empresas certificadas com a ISSO 14001. In: X SIMPÓSIO DE ENGENHARIA DE PRODUÇÃO, Anais..., Bauru, São Paulo, Brasil, 2003.

CARLSON, R.E. More complications in the chlorophyll-Secchi disk relationship. Limnology and Oceanography, EUA, v. 25, n. 2, p. 379382, 1980

CAMARGO, A.L.B. Desenvolvimento sustentável: dimensões e desafios. Campinas: Papirus, 2003.

CAPRA, F. A teia da vida: uma nova compreensão científica dos sistemas vivos. São Paulo: Cultrix, 1996.
CSILLAG, J.M. Análise do valor. São Paulo: Atlas, 1995.

CUNHA, F.L.S.J. Indicadores de sustentabilidade. In: VIII ENCONTRO NACIONAL SOBRE GESTÃO EMPRESARIAL E MEIO AMBIENTE, Anais..., Rio de Janeiro, 2005.

DEPONTI, C.M.; ECKERT, C.; AZAMBUJA, J.L.B. Estratégia para construção de indicadores para avaliação da sustentabilidade e monitoramento de sistemas. Agroecologia e Desenvolvimento Rural Sustentável, Porto Alegre, v. 3, n. 4, p. 44-52, out./dez. 2002.

DONAIRE, D. A utilização do estudo de casos como método de pesquisa na área da administração. Revista IMES, Ano XIV, n. 40, p. 9-19, 1997.

GIL, A.C. Técnicas de pesquisa em economia e elaboração de monografias. São Paulo: Atlas, 2002.

MEGER, D. G. Caracterização de material particulado inorgânico no reservatório do rio Passaúna. Dissertação (Mestrado em Gestão Ambiental) - Universidade Positivo, Curitiba, Paraná, 2007.

QUIROGA M.R. Estatísticas del médio ambiente em América Latina y el Caribe: avances y perspectivas. Chile: Cepal, 2005. Series Manuales.

RUFINO, R. C. Avaliação da qualidade ambiental do município de Tubarão (SC) através do uso de indicadores ambientais. 123 f. Dissertação (Mestrado em Engenharia de Produção) - Programa de Pós-Graduação em Engenharia de Produção, Florianópolis, 2002.

SANEPAR. Relatório da APA do Passaúna. Paraná, 2001.

VIEIRAS, X; POULIQUEN, X.; SOTO, M. 12 indicadores ambientais: Galiza insustentável. Santiago de Compostela: Asociación para a defensa Ecolóxica de Galiza (ADEGA), 2005. 